\title{
Inter-scan Reproducibility of Cardiovascular Magnetic Resonance Imaging-Derived Myocardial Perfusion Reserve Index in Women with no Obstructive Coronary Artery Disease
}

\author{
Ahmed Al-Badri ${ }^{1}$, Janet Wei ${ }^{1}$, Sofy Landes ${ }^{1}$, Manish Motwani ${ }^{3}$, Galen Cook-Wiens ${ }^{2}$, Michael D Nelson ${ }^{1}$, Puja K \\ Mehta $^{1}$, Chrisandra Shufelt ${ }^{1}$, Behzad Sharif ${ }^{3}$, Debiao Li ${ }^{3}$, Daniel S Berman ${ }^{3}$, Louise EJ Thomson and C Noel Bairey \\ $\operatorname{Merz}^{1 *}$
}

${ }^{1}$ Cedars Sinai Heart Institute, USA

${ }^{2}$ Biostatistics and Bioinformatics Research Center, Cedars-Sinai Medical Center, USA

${ }^{3} S$ Mark Taper Foundation Imaging Center, Cedars-Sinai Medical Center, USA

Received date: February 02, 2018; Published date: February 23, 2018

*Corresponding author: C Noel Bairey Merz, 127 S. San Vicente Blvd, Suite A3600, Los Angeles, CA 90048, Tel: 310-423-9680; Fax: 310-4239681; Email: Noel.BaireyMerz@cshs.org

\begin{abstract}
Background: Cardiovascular magnetic resonance imaging (CMRI) derived myocardial perfusion reserve index (MPRI) has recently been shown to detect coronary microvascular dysfunction (CMD) in women with signs and symptoms of ischemia and no obstructive coronary artery disease (CAD). The aim of this study was to determine the inter-scan reproducibility of MPRI in this patient group in order to assess its diagnostic robustness in serial scans and assess its utility as a marker of potential therapies for CMD.

Methods: Rest/stress perfusion CMR was performed at $1.5 \mathrm{~T}$ using a standardized protocol in 17 women with signs and symptoms of ischemia and no obstructive CAD on two separate days (within 90 days of each other). The same pharmacological stress agent (adenosine/ regadenoson) was used for both scans. MPRI was calculated from time-intensity curves of the whole myocardium and blood pool at stress and rest. One experienced observer, blinded to clinical data, performed all measurements. Intra-class correlation coefficients (ICC), coefficient of variation ( $\mathrm{CoV}$ ), and Bland-Altman plots were determined.
\end{abstract}

Results: Mean age was $53 \pm 10$ years old and BMI $28 \pm 7 \mathrm{~kg} / \mathrm{m} 2 ; 47 \%$ had hypertension, $4 \%$ diabetes, $9 \%$ hyperlipidemia and $10 \%$ family history of CAD. Mean MPRI for the 17 women was higher for scan 2 compared to scan 1 ( $1.98 \pm 0.3$ vs. $1.65 \pm 0.78$, respectively, p<0.001); and this relationship persisted even when corrected for resting rate pressure product (RPP) (2.42 \pm 0.81 vs. $1.97 \pm 0.92$, respectively, 0.002$)$. The mean bias for MPRI between sequential scans was 0.34 (95\% CI: 0.18 to 0.49 , limits of agreement: $-0.31,0.98$ and when corrected for resting RPP it was 0.45 ( $95 \%$ CI: 0.21 to 0.68 , limits of agreement: $-0.52,1.41)$. ICC and CoV also indicated modest inter-scan reproducibility (ICC 0.57; $\operatorname{CoV} 20.3 \%$ ), but both measures were comparable to values seen in prior studies in CAD populations and healthy volunteers.

Conclusion: Inter-scan reproducibility of CMRI-derived MPRI in women with suspected CMD is modest, with relatively wide limits of agreement. This variability is similar to that seen in other populations, suggesting that some caution must be exercised when using absolute MPRI cut-offs in isolation for the diagnosis of CMD or repeated measures of MPRI to track response to therapy. Additional work is ongoing to improve reproducibility from both biological and technological standpoints.

Keywords: Cardiac magnetic resonance imaging; Myocardial perfusion reserve index; Inter-scan; Reproducibility

Abbreviations: CMRI: Cardiac Magnetic Resonance Imaging; CAD: Coronary Artery Disease; ICC: Intra-Class Correlation Coefficients; CoV: Coefficient of Variation; RPP: Rate Pressure Product; PET: Positron Emission Tomography

\section{Introduction}

First-pass stress perfusion cardiac magnetic resonance imaging (CMRI) can detect vasodilator stress-induced myocardial hypo-perfusion in patients with coronary artery disease (CAD) with high sensitivity and specificity $[1,2]$. Women with signs and symptoms of ischemia but no obstructive CAD are an increasingly recognized patient group requiring more investigational study to understand the best diagnostic and management strategies. These patients often have coronary micro vascular dysfunction (CMD) which is associated with cardiovascular adverse outcomes [1,2]. Invasive coronary reactivity testing is the reference standard for diagnosing CMD using different vasoactive medications 
to evaluate the endothelial and non-endothelial-dependent coronary function. Increased rates of cardiac death, stroke, and new onset heart failure have been observed during 4.5 years follow up among women with reduced invasive coronary flow reserve (CFR) to adenosine [3].

CMRI is noninvasive imaging technique that can be used to diagnose CMD through assessment of rest and stress myocardial perfusion [4]. Semi-quantitative analysis of the firstpass perfusion CMRI data can be used to calculate myocardial perfusion reserve index (MPRI), which is an indexed ratio of perfusion time intensity curves, as a measure of response to vasodilator stress, while positron emission tomography (PET) has a well-established evidence-base for robust non-invasive CFR assessment. CMRI has the advantage of the lack of radiation which is particularly relevant in the predominantly young women who comprise this patient group. This advantage would be of particular importance for the use of serial imaging to monitor treatment response. In the context of serial studies, the inter-scan reproducibility of CMRI derived MPRI measurement needs to be established, so that differences between groups or values obtained in individuals can be defined as being real or potentially due to known variation in measurement. Therefore, the aim of this study was to assess the inter-study agreement of semi-quantitative MPRI in women with signs and symptoms of ischemia and no obstructive CAD undergoing serial stress perfusion CMRI.

\section{Methods}

\section{CMRI procedures}

Seventeen women underwent 2 serial rest/stress perfusion CMRI scans within 90 days of each other as part of their participation in the RWISE study [5]. The study was an IRB approved clinical trial under the care of the Women's Heart Center, Cedars-Sinai Heart Institute, Los Angeles, California and university of Florida, Gainesville, FL (NCT01342029). Accordingly, all subjects had been referred for evaluation of signs and symptoms of ischemia with no obstructive CAD (defined as $<50 \%$ luminal diameter stenosis of epicardial coronary artery on invasive coronary angiography). Subjects with significant CAD (epicardial artery stenosis $\geq 50 \%$ ), coronary artery anomalies, and visible coronary vasospasm during angiography or bridging were excluded.

Therefore, data from a total of 34 scans performed were available for analysis. The paired scans were performed with the same vasodilator stress agent i.e. using adenosine or regadenoson. All studies were performed between May 2011 and September 2015. All subjects had evidence of CMD confirmed by either invasive coronary reactivity testing (defined as CFR $<2.5$, or no dilatation [ $\leq 0 \%$ change] in response to acetylcholine) [6] as part of clinical care or stress CMRI (defined as MPRI $<2.0$ ). All vasoactive medications were stopped at least 24 hours before CMR testing as per protocol [5]. The rest/stress CMRI was performed following the previously published protocol [4].

\section{CMR quantitative analysis}

CMRI data were interpreted by one expert reader experienced in performance and interpretation of CMRI (L.E.J. Thomson) blinded to clinical data. Semi- quantitative analysis of the first pass perfusion images was performed using CAAS MRV 3.3 software (Pie Medical Imaging B.V., Netherlands). The endocardial and epicardial contours were manually defined and adjusted, frame by frame if needed, to optimize sampling of the myocardium. Care was taken to exclude blood pool activity and to exclude any linear dark rim artifact at the LV cavity/endocardial border. The LV cavity region of interest was manually adjusted to include the region of maximal signal intensity within the cavity and to exclude papillary muscle. The reader manually defined the starting point (T0 cycle) and the ending point ( $\mathrm{T}$ end) of time intensity curves. T0 was set at the baseline point immediately prior to the upslope and $\mathrm{T}$ end was placed at the point where myocardial peak intensities were reached (Figure 1). The ratio of the maximum upslope of the selected curve, which corresponds to the specific myocardial segment, over the maximum upslope of the LV cavity curve, gives the relative upslope (RU). MPRI is then calculated by RU at stress divided by RU at rest. Data is generated by the software for subendocardial, subepicardial and transmural MPRI using standard AHA myocardial segmentation (16 segments due to absence of data for an apical segment) [7].

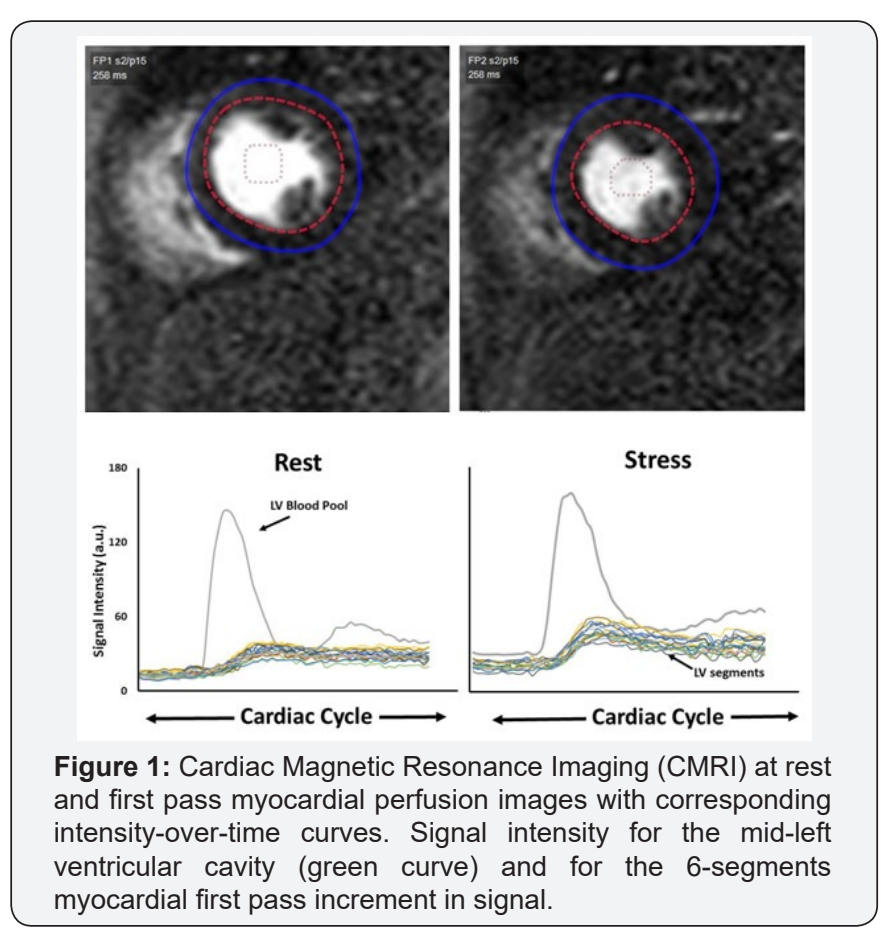

\section{Statistical analysis}

Variables were summarized by means and standard deviations, or counts and percentages if categorical. The ICC was calculated as the proportion of between subject variance from a linear regression model with only a fixed intercept effect and random subject intercepts. Coefficient of variation is reported as a percent of the standard deviation divided by the mean. An 


\section{Current Trends in Clinical \& Medical Imaging}

analysis of measurement agreement was carried out as described in Bland and Altman [8]. Significance for hypothesis tests was set at a level of 0.05 . All analyses were done using SAS version 9.3 (SAS Institute, Cary, NC) and R software. The MPRI was corrected to rate pressure product: Corrected MPRI: MPRI/rest RPP *104 if there is variability in heart rate and systolic blood pressure between scans for each subject [9].

\section{Results}

Subject characteristics are shown in Table 1 for the whole group. Mean age was $53 \pm 10$ years old and BMI $28 \pm 7 \mathrm{~kg} / \mathrm{m} 2$; $47 \%$ had hypertension, $4 \%$ diabetes, $9 \%$ hyperlipidemia and $10 \%$ family history of CAD. No significant complications occurred and all subjects completed the imaging protocol. The MPRI results are shown in Table 2. MPRI data is presented for the transmural (16 segment mean) as well as individual slices for both subendocardial and subepicardial regions. Inter-scan reproducibility of the left ventricular MPRI calculated by ICC and expressed as $\mathrm{CoV}$.

Table1: Demographic and Clinical characteristics $(n=17)$.

\begin{tabular}{|c|c|}
\hline Age (years) & $53 \pm 10$ \\
\hline $\operatorname{BMI}\left(\mathrm{kg} / \mathrm{m}^{2}\right)$ & $28 \pm 7$ \\
\hline Hypertension & $8(47 \%)$ \\
\hline Diabetes & $4(24 \%)$ \\
\hline Hyperlipidemia & $9(53 \%)$ \\
\hline $\begin{array}{c}\text { Family history of premature coronary artery } \\
\text { disease }\end{array}$ & $10(59 \%)$ \\
\hline Current smoker & $0 \%$ \\
\hline Former Smoker & $4(24 \%)$ \\
\hline \multicolumn{2}{|l|}{ Symptoms } \\
\hline Typical angina & $9(53 \%)$ \\
\hline Shortness of breath & $13(77 \%)$ \\
\hline Palpitations & $8(47 \%)$ \\
\hline Nausea & $4(23.5 \%)$ \\
\hline \multicolumn{2}{|l|}{ Medications } \\
\hline - Beta blockers & $9(53 \%)$ \\
\hline - Calcium channel blockers & $8(47 \%)$ \\
\hline - ACEI or ARB & $(29 \%)$ \\
\hline - Nitrates & $13(77 \%)$ \\
\hline - HMG-CoA reductase inhibitors & $10(59 \%)$ \\
\hline - Aspirin & $14(82 \%)$ \\
\hline - Diuretic & $2(12 \%)$ \\
\hline
\end{tabular}

BMI: Basal metabolic index, ACEI: angiotensin-converting enzyme inhibitors, ARB: angiotensin receptor blockers
Table 2: Inter-scan reproducibility of post processing MPRI* $(N=17)$.

\begin{tabular}{|c|c|c|c|c|}
\hline & Scan 1 & Scan 2 & $\mathrm{CoV}$ & ICC \\
\hline \multicolumn{5}{|c|}{ All Slices } \\
\hline $\begin{array}{l}\text { Mean whole MPRI } \\
\text { segments 1-16 }\end{array}$ & $1.97 \pm 0.92$ & $2.42 \pm 0.81$ & $20.30 \%$ & 0.57 \\
\hline $\begin{array}{c}\text { Mean } \\
\text { subendocardial } \\
\text { MPRI segments } \\
1-16\end{array}$ & $1.81 \pm 0.82$ & $2.13 \pm 0.64$ & $16.20 \%$ & 0.52 \\
\hline $\begin{array}{c}\text { Mean } \\
\text { subepicardial } \\
\text { MPRI segments } \\
1-16\end{array}$ & $2.1 \pm 1.10$ & $2.56 \pm 0.91$ & $20.70 \%$ & 0.56 \\
\hline \multicolumn{5}{|c|}{ Basal Slices } \\
\hline $\begin{array}{l}\text { Mean whole MPRI } \\
\text { segments 1-6 }\end{array}$ & $1.98 \pm 0.90$ & $2.31 \pm 0.82$ & $15.70 \%$ & 0.56 \\
\hline $\begin{array}{c}\text { Mean } \\
\text { subendocardial } \\
\text { MPRI segments } \\
1-6\end{array}$ & $1.88 \pm 0.82$ & $2.06 \pm 0.70$ & $9.70 \%$ & 0.51 \\
\hline $\begin{array}{c}\text { Mean } \\
\text { subepicardial } \\
\text { MPRI segments } \\
1-6\end{array}$ & $2.02 \pm 1.01$ & $2.51 \pm 0.96$ & $21.80 \%$ & 0.43 \\
\hline \multicolumn{5}{|c|}{ Mid Slices } \\
\hline $\begin{array}{l}\text { Mean whole MPRI } \\
\text { segments } 7-12\end{array}$ & $2.03 \pm 1.01$ & $2.59 \pm 0.96$ & $24.40 \%$ & 0.55 \\
\hline $\begin{array}{c}\text { Mean } \\
\text { subendocardial } \\
\text { MPRI segments } \\
7-12\end{array}$ & $1.74 \pm 0.84$ & $2.25 \pm 0.78$ & $25.60 \%$ & 0.47 \\
\hline $\begin{array}{c}\text { Mean } \\
\text { subepicardial } \\
\text { MPRI segments } \\
7-12\end{array}$ & $2.24 \pm 1.29$ & $2.69 \pm 1.02$ & $18.50 \%$ & 0.60 \\
\hline \multicolumn{5}{|c|}{ Apical Slices } \\
\hline $\begin{array}{l}\text { Mean whole MPRI } \\
\text { segments } 13-16\end{array}$ & $1.94 \pm 1.02$ & $2.39 \pm 0.75$ & $17.40 \%$ & 0.54 \\
\hline $\begin{array}{c}\text { Mean } \\
\text { subendocardial } \\
\text { MPRI segments } \\
13-16\end{array}$ & $1.88 \pm 0.96$ & $2.13 \pm 0.62$ & $7.80 \%$ & 0.57 \\
\hline $\begin{array}{c}\text { Mean } \\
\text { subepicardial } \\
\text { MPRI segments } \\
13-16\end{array}$ & $1.99 \pm 1.13$ & $2.54 \pm 0.96$ & $21.70 \%$ & 0.54 \\
\hline
\end{tabular}

MPRI: Myocardial Perfusion Reserve Index, CoV: Coefficient of Variation, ICC: Intra-Class Correlation Coefficient

*MPRI was corrected to the resting rate pressure product

Mean MPRI was higher for scan 2 compared to scan 1 $(1.98 \pm 0.3$ vs. $1.65 \pm 0.78$, respectively, $\mathrm{p}<0.001)$; and this relationship persisted even when corrected for resting RPP $(2.42 \pm 0.81$ vs. $1.97 \pm 0.92$, respectively, $\mathrm{p}<0.002)$. The average difference for MPRI between scans was 0.34 (95\% CI: 0.18 to 0.49$)$ and for resting RPP corrected MPRI it was $0.45(95 \%$ CI: 0.21 to 0.68 ). The result of Bland-Altman analysis for transmural and mid-ventricular MPRI is shown in Figure $2 \& 3$, 
respectively. The limits of agreement for the 0.34 bias in MPRI reproducibility were $(-0.31,0.98)$ and for the 0.45 bias in resting RPP corrected MPRI reproducibility they were $(-0.52,1.41)$. The subendocardial MPRI for all slices was more reproducible (CoV 16.2) compared to mean whole MPRI (CoV 20.3) and mean subepicardial MPRI (CoV 20.7 \%). There was a modest inter-scan ICC $0.57,0.52$ and 0.57 within transmural, subendocardial and subepicardial myocardial regions respectively. The CoV was $20 \%$ for the transmural MPRI.

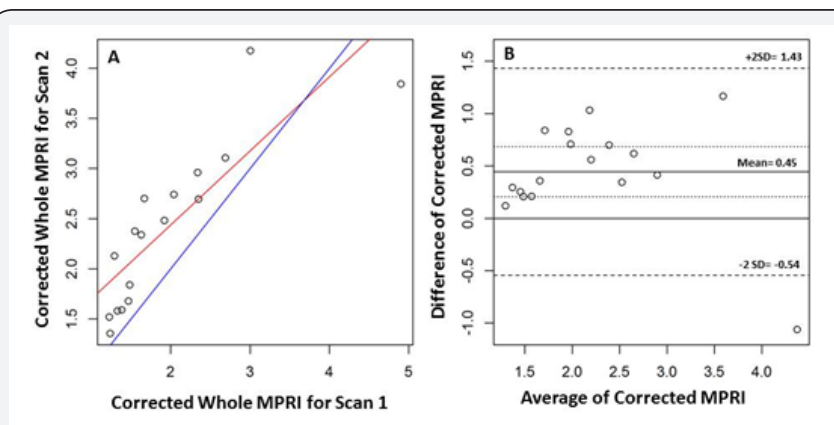

Figure 2: Agreement of Whole Myocardial Perfusion Reserve Index (MPRI) between Scan 1 and Scan 2. Linear regression graph (A) and Bland-Altman plot (B).
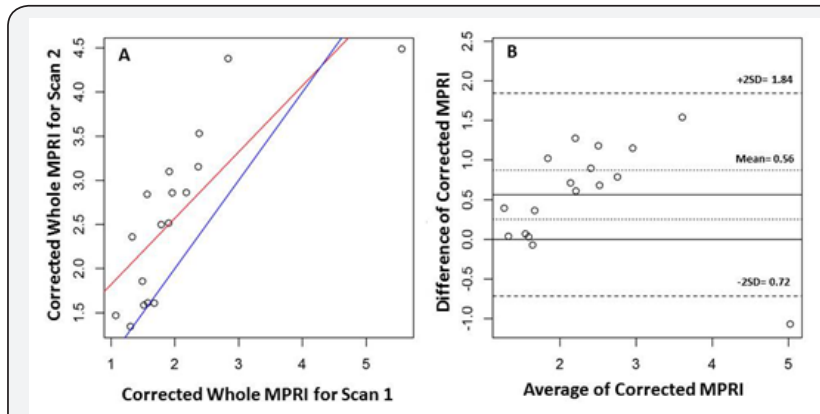

Figure 3: Agreement of Mid-ventricular Myocardial Perfusion Reserve Index (MPRI) between Scan 1 and Scan 2. Linear regression graph $(A)$ and Bland-Altman plot $(B)$.

\section{Discussion}

In our study, first pass myocardial perfusion CMRI for semiquantitative analysis of MPRI measurements on serial CMRI studies, using commercially available software, was shown to have modest inter-scan reproducibility. The inter-scan reproducibility of CMRI-derived MPRI in the novel population of women with suspected CMD was modest, with relatively wide limits of agreement. There is limited published data available for the reproducibility of serial myocardial perfusion measurements using CMRI. Inter-study reproducibility of CMRI has been reported for LV volumes, ejection fraction and mass [9-11], and there is limited data describing the reproducibility of semiquantitative analysis of stress perfusion CMR. We have previously defined reproducibility of repeated MPRI calculation for CAAS MRV 3.3 software (Pie Medical Imaging B.V., Netherlands) and found intra-observer coefficient of variation 3.6\%, inter-observer coefficient of variation $7.5 \%$ (12). The inter-scan reproducibility of CMRI myocardial stress perfusion has been reported using visual, semi-quantitative and fully quantitative approaches. Larghat et al. [12] studied the reproducibility of myocardial perfusion CMRI in 11 normal subjects (including 5 women) during adenosine stress and rest on 2 separate days with mean interscan delay of 84 days (range 7-280 days). Imaging was at 1.5T (Philips Medical Systems) and post processing used Q Mass 6.1.6, Medis. The coefficient of variation ( $\mathrm{CoV}$ ) was $19.4 \%$ for semi-quantitative inter-study comparison and $27 \%$ for fully quantitative analysis of myocardial perfusion reserve. Chih et al. [9] evaluated twenty subjects (10 with CAD and 10 controls at low Framingham risk of CAD, including 4 women). Imaging was at 1.5T (Philips Medical Systems) and post processing used Philips View Forum workstation. The CoV for visual (qualitative) segmental analysis of patients was $30.6 \%$. The CoV for global MPRI was $23 \%$ for patients with CAD and $18 \%$ in the control group. They also reported software based reproducibility data with intra-observer CoV of 5.3\%, inter-observer CoV 9.0\%.

PET is a well-established method for non-invasive quantitation of myocardial flow and prior studies have shown variability in repeated measurement of hyperemic flow using absolute quantitative approaches with both radio labeled water and ammonia. Kauffman et al studied 21 normal volunteers (number of women not reported) with 0 labeled water with two assessments within one hour and reported repeatability coefficient (smallest real difference) for global measurement of coronary vasodilator reserve of 1.32 (33\% of mean) [13]. Nagamachi et al. [14] used [13] N-ammonia PET with repeated measurements in volunteers (including 4 women) and reported variation in both rest and stress absolute flow measurements repeated same day $(n=8)$ or different day $(n=13)$. Differences were normalized to rate pressure product, with reported two day mean percentage difference in absolute vasodilator stress perfusion $10.3 \pm 10.5 \%$. More recently, repeated measurement of coronary flow reserve using 82Rubidium PET reported a mean difference of $-4.14 \pm 18 \%$ between first and second measures performed 60 minutes apart in 15 healthy volunteers (including 7 women) [15].

In our data, there were wide limits of agreement for serial MPRI measurement derived from CMR (Figure 2). This emphasizes the need for caution in over interpreting small changes in MPRI between repeated studies. There are multiple factors that potentially contribute to variation in myocardial perfusion reserve measurement. By protocol, the two scans were performed at the same time of day, with identical pre-test preparation (including medication and caffeine withdrawal), scanner hardware and software settings and pharmacologic stress agent. The influence of resting hemodynamic state was adjusted for in statistical analysis. Variation related to post processing of data was minimized by use of a single observer.

The main limitation of this study is the retrospective nature. Thus, there might be a change in subjects' disease process, medications and clinical status between the scans. However, we tried to select subjects whose their medications and clinical 


\section{Current Trends in Clinical \& Medical Imaging}

status unchanged between the two scans. Due to the latter, small number of subjects were included in this analysis. Yet, we believe the study is adequately powered to examine test-retest reproducibility, our sample is larger than the prior reports, and represents a first evaluation in women with microvascular dysfunction. Another limitation of our study is the length of time between scans (90 days). However in previous reproducibility study evaluated myocardial perfusion index using SPECT, the repeat scan was performed 9-22 months after the first one. The study showed that myocardial perfusion index was highly correlated and reproducible [16]. While we detected only a modest difference between test visits, there was a bias to increase in MRRI on the second compared to the first visit. Patients were being clinically managed between the two visits, making it possible that the improvement bias reflects 'real' change, perhaps due to use of medications such as HMG-CoA reductase inhibitors, ACE inhibitors and angiotensin receptor blockers that could have influenced microvascular function but were not withdrawn prior to stress testing [17]. Our study did not include normal subjects, though this is more closely aligns with clinical practice as patients with normal test are less likely to undergo serial testing. We believe more work in this area is needed.

\section{Conclusion}

Inter-scan reproducibility of CMRI-derived MPRI in women with suspected CMD is modest, with relatively wide limits of agreement. This variability is similar to that seen in other populations, and is comparable to PET, suggesting that some caution must be exercised when using absolute MPRI cut-offs in isolation for the diagnosis of CMD, or repeated measures of MPRI to track response to therapy. Additional work is ongoing to improve reproducibility and understand sources of variation of MPRI measurement from both biological and technological standpoints.

\section{Acknowledgement}

This work was supported by contracts from the National Heart, Lung and Blood Institutes nos. N01-HV-68161, N01-HV-68162, N01-HV-68163, N01-HV-68164, grants U0164829, U01 HL649141, U01 HL649241, K23HL105787, T32HL69751, R01 HL090957, 1R03AG032631 from the National Institute on Aging, GCRC grant M01-RR00425 from the National Center for Research Resources, the National Center for Advancing Translational Sciences Grant UL1TR000124 and UL1TR000064, and grants from the Gustavus and Louis Pfeiffer Research Foundation, Danville, NJ, The Women's Guild of Cedars-Sinai Medical Center, Los Angeles, CA, The Ladies Hospital Aid Society of Western Pennsylvania, Pittsburgh, PA, and QMED, Inc., Laurence Harbor, NJ, the Edythe L. Broad and the Constance Austin Women's Heart Research Fellowships, Cedars-Sinai Medical Center, Los Angeles, California, the Barbra Streisand Women's Cardiovascular Research and Education Program, Cedars-Sinai Medical Center, Los Angeles, The Society for Women's Health Research (SWHR),
Washington, D.C., The Linda Joy Pollin Women's Heart Health Program, and the Erika Glazer Women's Heart Health Project, Cedars-Sinai Medical Center, Los Angeles, California.

\section{References}

1. Johnson BD, Shaw LJ, Buchthal SD, Bairey Merz CN, et al. (2004) Prognosis in women with myocardial ischemia in the absence of obstructive coronary disease: results from the National Institutes of Health-National Heart, Lung, and Blood Institute-Sponsored Women's Ischemia Syndrome Evaluation (WISE). Circulation 109(24): 2993-2999.

2. von Mering GO, Arant CB, Wessel TR, McGorray SP, Merz BCN, et al. (2004) Abnormal coronary vasomotion as a prognostic indicator of cardiovascular events in women: results from the National Heart, Lung, and Blood Institute-Sponsored Women's Ischemia Syndrome Evaluation (WISE). Circulation 109(6): 722-775.

3. Pepine CJ, Anderson RD, Sharaf BL, Reis SE, Smith KM, et al. (2010) Coronary microvascular reactivity to adenosine predicts adverse outcome in women evaluated for suspected ischemia results from the National Heart, Lung and Blood Institute WISE (Women's Ischemia Syndrome Evaluation) study. J Am Coll Cardiol 55(25): 2825-2832.

4. Thomson LE, Wei J, Agarwal M, Haft-Baradaran A, Shufelt C, et al. (2015) Cardiac magnetic resonance myocardial perfusion reserve index is reduced in women with coronary microvascular dysfunction. A National Heart, Lung, and Blood Institute-sponsored study from the Women's Ischemia Syndrome Evaluation. Circ Cardiovasc imaging 8(4).

5. Bakir M, Wei J, Nelson MD, Mehta PK, Haftbaradaran A, et al. (2016) Cardiac magnetic resonance imaging for myocardial perfusion and diastolic function-reference control values for women. Cardiovasc Diagn Ther 6(1): 78-86.

6. Wei J, Mehta PK, Johnson BD, Samuels B, Kar S, et al. (2012) Safety of coronary reactivity testing in women with no obstructive coronary artery disease: results from the NHLBI-sponsored WISE (Women's Ischemia Syndrome Evaluation) study. JACC Cardiovasc Interv 5(6): 646-653.

7. Hundley WG, Bluemke DA, Finn JP, Flamm SD, Fogel MA, et al. (2010) ACCF/ACR/AHA/NASCI/SCMR 2010 expert consensus document on cardiovascular magnetic resonance: a report of the American College of Cardiology Foundation Task Force on Expert Consensus Documents. Circulation 121: 2462-2508.

8. Bland JM, Altman DG (1999) Measuring agreement in method comparison studies. Statistical methods in medical research 8(2): 135-160.

9. Chih S, Macdonald PS, Feneley MP, Law M, Graham RM, et al. (2010) Reproducibility of adenosine stress cardiovascular magnetic resonance in multi-vessel symptomatic coronary artery disease. J Cardiovasc Magn Reson 12: 42.

10. Grothues F, Smith GC, Moon JC, Bellenger NG, Collins P, et al. (2002) Comparison of interstudy reproducibility of cardiovascular magnetic resonance with two-dimensional echocardiography in normal subjects and in patients with heart failure or left ventricular hypertrophy. Am J Cardiol 90(1): 29-34.

11. Bellenger NG, Burgess MI, Ray SG, Lahiri A, Coats AJ, et al. (2000) Comparison of left ventricular ejection fraction and volumes in heart failure by echocardiography, radionuclide ventriculography and cardiovascular magnetic resonance; are they interchangeable? Eur Heart J 21(16): $1387-1396$

12. Larghat AM, Maredia N, Biglands J, Greenwood JP, Ball SG, et al. (2013) Reproducibility of first-pass cardiovascular magnetic resonance myocardial perfusion. Journal of magnetic resonance imaging 37(4): 865874.

13. Kaufmann PA, Gnecchi-Ruscone T, Yap JT, Rimoldi O, Camici PG (1999) Assessment of the reproducibility of baseline and hyperemic myocar- 


\section{Current Trends in Clinical \& Medical Imaging}

dial blood flow measurements with 150-labeled water and PET. J Nucl Med 40(11): 1848-1856.

14. Nagamachi S, Czernin J, Kim AS, Sun KT, Bottcher M, et al. (1996) Reproducibility of measurements of regional resting and hyperemic myocardial blood flow assessed with PET. J Nucl Med 37(10): 1626-1631.

15. Manabe O, Yoshinaga K, Katoh C, Naya M, deKemp RA, et al. (2009) Repeatability of rest and hyperemic myocardial blood flow measurements with 82Rb dynamic PET. J Nucl Med 50(1): 68-71.

16. Berman DS, Kang X, Gransar H, Gerlach J, Friedman JD, et al. (2009) Quantitative assessment of myocardial perfusion abnormality on
SPECT myocardial perfusion imaging is more reproducible than expert visual analysis. J Nucl Cardiol 16(1): 45-53

17. Pauly DF, Johnson BD, Anderson RD, Handberg EM, Smith KM, et al. (2011) In women with symptoms of cardiac ischemia, nonobstructive coronary arteries, and microvascular dysfunction, angiotensin-converting enzyme inhibition is associated with improved microvascular function: A double-blind randomized study from the National Heart, Lung and Blood Institute Women's Ischemia Syndrome Evaluation (WISE). Am Heart J 162(4): 678-684.

\section{Your next submission with Juniper Publishers} will reach you the below assets

- Quality Editorial service

- Swift Peer Review

- Reprints availability

- E-prints Service

- Manuscript Podcast for convenient understanding

- Global attainment for your research

- Manuscript accessibility in different formats

( Pdf, E-pub, Full Text, Audio)

- Unceasing customer service

Track the below URL for one-step submission https://juniperpublishers.com/online-submission.php 